\title{
Targeting myeloid-inflamed tumor with anti-CSF-1R antibody expands CD137+ effector T-cells in the murine model of pancreatic cancer
}

May Tun Saung 1,2,8,9, Stephen Muth 1,2,8,9, Ding Ding ${ }^{1,2,8,9}$, Dwayne L. Thomas $\|^{1,2,8,9}$, Alex B. Blair ${ }^{2,3,8,9}$,

Takahiro Tsujikawa ${ }^{4,5}$, Lisa Coussens ${ }^{5,6}$, Elizabeth M. Jaffee ${ }^{1,2,7,8,9}$ and Lei Zheng ${ }^{1,2,3,7,8,9^{*}}$ (D)

\begin{abstract}
Background: The pancreatic cancer vaccine, GVAX, induces novel lymphoid aggregates in the otherwise immune quiescent pancreatic ductal adenocarcinoma (PDAC). GVAX also upregulates the PD-1/PD-L1 pathway, and a preclinical model demonstrated the anti-tumor effects of combination GVAX and anti-PD-1 antibody therapy (GVAX/ aPD-1). Resistance to GVAX was associated with an immune-suppressive myeloid cell infiltration, which may limit further therapeutic gains of GVAX/aPD-1 therapy. The expression of CSF-1R, a receptor important for myeloid cell migration, differentiation and survival, and the effect of its therapeutic blockade in the context of GVAX in PDAC has not been investigated.
\end{abstract}

Methods: Lymphoid aggregates appreciated in 24 surgically resected PDAC from patients who received one dose of neoadjuvant GVAX were analyzed with multiplex immunohistochemistry. Flow cytometry analysis of tumor infiltrating T-cells in a murine model of PDAC was performed to investigate the therapeutic effects and mechanism of anti-CSF-1R/anti-PD-1/GVAX combination immunotherapy.

Results: High CSF-1R expression in resected PDAC from patients who received neoadjuvant GVAX was associated with a higher myeloid to lymphoid cell ratio $(p<0.05)$, which has been associated with poorer survival. This higher CSF-1R expression was associated with a higher intra-tumoral infiltration of immature dendritic cells $(p<0.05)$, but not mature dendritic cells $(p=0.132)$. In the pre-clinical murine model, administering anti-CSF-1R antibody prior to and after GVAX/aPD-1 ("pre/post-aCSF-1R + aPD-1 + GVAX") enhanced the survival rate compared to GVAX/aPD-1 dual therapy $(p=0.005)$, but administering anti-CSF-1R only before GVAX/aPD-1 did not $(p=0.41)$. The "pre/postaCSF-1R + aPD-1 + GVAX" group also had higher intra-tumoral infiltration of PD-1 + CD8+ and PD-1 + CD4+ T-cells compared to aPD-1/GVAX $(p<0.001)$. Furthermore, this regimen increased the intra-tumoral infiltration of PD-1 + CD137+ CD8+, PD-1 + CD137 + CD4+ and PD-1 + OX40 + CD4+ T-cells $(p<0.001)$. These PD-1 + CD137 + CD8+ Tcells expressed high levels of interferon- $\gamma$ (median 80-90\%) in response to stimulation with CD3/CD28 activation beads, and this expression was higher than that of PD-1 + CD137-CD8+ T-cells $(p<0.001)$.

(Continued on next page)

\footnotetext{
* Correspondence: Izheng6@jhmi.edu

'Department of Oncology, Johns Hopkins University School of Medicine,

Baltimore, MD, USA

${ }^{2}$ The Sidney Kimmel Cancer Center, Johns Hopkins University School of

Medicine, Baltimore, MD, USA

Full list of author information is available at the end of the article
}

(c) The Author(s). 2018 Open Access This article is distributed under the terms of the Creative Commons Attribution 4.0 International License (http://creativecommons.org/licenses/by/4.0/), which permits unrestricted use, distribution, and

reproduction in any medium, provided you give appropriate credit to the original author(s) and the source, provide a link to the Creative Commons license, and indicate if changes were made. The Creative Commons Public Domain Dedication waiver (http://creativecommons.org/publicdomain/zero/1.0/) applies to the data made available in this article, unless otherwise stated. 
(Continued from previous page)

Conclusions: The conversion of exhausted PD-1+ T-cells to CD137+ activated effector T-cells may contribute to the anti-tumor effects of the anti-CSF-1R/anti-PD-1/GVAX combination therapy. Anti-CSF-1R antibody with anti-PD-1 antibody and GVAX have the potential be an effective therapeutic strategy for treatment of PDAC.

Keywords: Pancreatic ductal adenocarcinoma, Lymphoid aggregates, Cytotoxic T-cells, Tumor associated macrophages, Dendritic cells, PD-1, CSF-1R, CD137, GVAX, Interferon- $\gamma$

\section{Background}

Pancreatic ductal adenocarcinoma (PDAC) is a devastating disease with a 5 -year survival rate of $8 \%$ for all stages despite the availability of treatment with chemotherapy, radiation and/or surgery [1]. The survival decreases to $3 \%$ for patients with late stage disease [1]. Immunotherapy has shown few clinical responses in PDAC despite clinical success in other cancers [2-5]. Resistance to immunotherapy has in part been attributed to an immune quiescent tumor microenvironment (TME). The presence of increased anti-tumor effector T-cells may improve prognosis, but these effectors cells are rarely appreciated in PDAC [6, 7]. Additionally, when infiltrating immune cells are present, they tend to be immunosuppressive, such as regulatory T-cells, immature dendritic cells, myeloid-derived suppressor cells (MDSCs) and tumor-associated macrophages (TAMs) [8].

To induce infiltration of immune cells into the PDAC, a GM-CSF (granulocyte-macrophage colony-stimulating factor) secreting pancreatic cancer vaccine, GVAX, has been employed [3, 4, 9-11]. A single dose of neoadjuvant GVAX with or without immunomodulatory doses of cyclophosphamide induced the formation of tertiary lymphoid structures within two weeks of administration in $85 \%$ of vaccinated patients, whereas organized lymphoid structures were not present in unvaccinated patients (ClinicalTrials.gov identifier: NCT007272441) [3]. These tertiary lymphoid structures had organized T-cell and B-cell zones, germinal centers, lymphatic vessels and the presence of cytokines involved in lymphoid neogenesis [3]. The presence of similar lymphoid structures in immunotherapy-naïve patients has been associated with improved survival, and indeed patients with an overall survival of over 3 years were more likely to have developed lymphoid aggregates after GVAX [3, 12]. However, there were still patients who survived less than 1.5 years despite having developed organized lymphoid structures after GVAX treatment [3]. Further analysis of these lymphoid structures with multiplex immunohistochemistry (IHC) demonstrated that patients with high myeloid cell infiltration had lower survival compared to those with low myeloid cell infiltration, despite high lymphoid cell densities in both groups [13]. High myeloid and CD68+ infiltration into tumors has been associated with poor survival in many studies [14-17]. Thus, targeting myeloid cells may further enhance anti-tumor activity of vaccine therapy. Of particular interest would be to target the colony-stimulating factor 1-receptor (CSF-1R) within this patient cohort. CSF-1 and its receptor, CSF-1R, regulate the migration, differentiation and survival of macrophages and other myeloid cells $[18,19]$. PDAC tumors often express high levels of CSF-1 compared to normal tissue, and CSF-1R can be detected in the tumor stroma [20, 21]. High CSF-1R expression in PDAC has also been associated with worse prognosis compared to low CSF-1R expression [20, 21]. Inhibition of CSF-1R can lead to preferential TAM depletion and modulate the remaining myeloid cells toward a more anti-tumor phenotype and away from pro-tumor properties [19-21]. The combination of anti-CSF-1R and anti-PD-1 antibodies promotes antitumor activity in pre-clinical models, and this combination is actively investigated in several clinical trials for many types of tumors, including pancreatic cancer [20, 22-24]. It is encouraging that one clinical trial has already reported that dual PD-1 and CSF-1R blockade may provide durable clinical benefit in patients with chemotherapy-treated and immunotherapy-naive advanced pancreatic cancer (ClinicalTrials.gov identifier: NCT02526017) [25].

In our prior study, GVAX induced both markers of early T-cell activation and PD-L1 and PD-1 expression within the lymphoid aggregates present in the post-vaccination patients, suggesting that vaccine therapy can prime PDAC for PD-1/PD-L1 inhibition therapies [3]. The survival benefit of adding GVAX to PD-1 blockade was demonstrated in a murine model of liver metastatic pancreatic cancer [26]. We propose that the addition of anti-CSF-1R antibody to the combination of GVAX therapy and anti-PD-1 antibody can improve the survival outcome of PDAC by further altering the myeloid population and creating a more anti-tumor immune environment.

\section{Study methods}

Human pancreatic ductal adenocarcinoma multiplex IHC The effect of CSF-1R expression level on the tumor-infiltrating immune cells in human PDAC tumor specimens was investigated based on the data generated from our previous multiplex immunohistochemistry analysis [13]. We analyzed 24 formalin-fixed paraffin-embedded specimens obtained after surgical resection of the tumor two weeks after one neoadjuvant intradermal administration of 
GVAX alone or in combination with immune modulatory doses of cyclophosphamide in patients with resectable PDAC (ClinicalTrials.gov identifier: NCT00727441) [3]. These 24 specimens had novel intratumoral lymphoid aggregates that were not present prior to GVAX administration [13]. The median cell density of CSF-1R expression was used as the cut off between high CSF-1R expression (higher than 14.5 cells $/ \mathrm{mm}^{2}$ ) and low CSF-1R expression (lower than $14.5 \mathrm{cell} / \mathrm{mm}^{2}$ ), and the effect the level of CSF-1R expression had on the infiltration of immune cells into the PDAC was analyzed. Lymphoid cells were CD45+ and CD3 + or CD20+ or CD56+, and myeloid markers were CD45+ and CD3-CD20-CD56- [13]. Within the myeloid cells, myelomonocytic cells were identified as CD66b-Tryptase-CD68 + CSF1R-, immature dendritic cells were identified as CD66b-Tryptase-MHC class II + DC-SIGN+CD83- and mature dendritic cells were identified as CD66b-Tryptase$\mathrm{MHC}$ class II + CD83+ [13]. All cell densities were measured as cells positive for marker per $\mathrm{mm}^{2}$. Cell densities (cells $/ \mathrm{mm}^{2}$ ) were used to calculated ratios between cell types.

\section{Cell lines and medium}

KPC is a previously established PDAC tumor cell line that was derived from transgenic mice in a C57Bl6 background with tissue-specific Kras and p53 knock-in mutations [27]. KPC cells were maintained at $37{ }^{\circ} \mathrm{C}$ in $5 \%$ $\mathrm{CO}_{2}$ with RMPI 1640 media (Life Technologies) supplemented with $10 \%$ heat-inactivated fetal bovine serum (HI-FBS, Benchmark), 1\% penicillin/streptomycin (pen/ strep, Life Technologies), 1\% MEM-NEAA, -(Minimum Essential Medium-Non-Essential Amino Acids, Life Technologies), $1 \%$ sodium pyruvate (Sigma) and 1\% L-glutamine (Life Technologies). B78H1 is an MHC (major histocompatibility complex) class I negative murine fibroblast cell line engineered to secrete GM-CSF. $\mathrm{B} 78 \mathrm{H} 1$ cells were maintained at $37{ }^{\circ} \mathrm{C}$ in $5 \% \mathrm{CO}_{2}$ with RMPI 1640 media supplemented with 10\% HI-FBS, 1\% pen/strep and $0.5 \% \%$ L-glutamine. Harvested tumor-infiltrating immune cells were processed in T-cell media, which consisted of RPMI 1640 media supplemented with $10 \%$ HI-FBS, $1 \%$ pen/strep, 1\% HEPES (Life Technologies), $1 \%$ MEM-NEAA, $0.5 \%$ L-glutamine and $0.05 \%$ 2-mercaptoethanol (Sigma).

\section{Mice tumor inoculation and treatment regimens}

Seven- to eight-week old C57Bl6 mice were purchased from Harlan Laboratories or Jackson Laboratories, and maintained in accordance with the Institutional Animal Care and Use Committee (IACUC) guidelines. When the mice were eight- to ten-weeks old, KPC tumor cell inoculation was performed via the hemispleen technique on day 0 as previously described [28]. Briefly, after anesthetizing the mice, the spleen was eviscerated, clipped and divided in half. $2 \times 10^{5} \mathrm{KPC}$ tumor cells in $100 \mu \mathrm{L}$ of PBS (Gibco) were carefully injected into half the spleen, and flushed with $150 \mu \mathrm{L}$ of PBS. The half of the spleen through which the tumor cells were injected was then removed to avoid leaving residual tumor cells external to the liver. The peritoneum and the skin were then sutured.

Anti-mouse PD-1 G1-D265A antibodies (clone 9H2, Bristol-Myers-Squibb) were administered intraperitoneally (IP) at $100 \mu \mathrm{g}(5 \mathrm{mg} / \mathrm{kg})$ per dose on days 7 and 11 , and murine GVAX vaccine was administered subcutaneously (SC) on day 7. GVAX vaccine was prepared by combining $1 \times 10^{6} \mathrm{KPC}$ cells and $1 \times 10^{6} \mathrm{~B} 78 \mathrm{H} 1$ cells in PBS to make a total combined cell concentration of $20 \times 10^{6}$ cells $/ \mathrm{mL}$. The cell suspension was then irradiated at 50 Gy and administered SC in 3 locations (bilateral flanks and one of the upper limbs) at $100 \mu \mathrm{L}$ per injection $\left(6 \times 10^{6}\right.$ cells injected in total per mouse). For "Pre/Post- $\alpha$ CSF-1R" dosing, rat anti-mouse CSF-1R G2a antibodies (clone AFS98, BioXcell) were administered IP at $1.5 \mathrm{mg}(75 \mathrm{mg} / \mathrm{kg})$ per dose on days $1,4,8$ and 11 . For "Pre- $\alpha$ CSF-1R" dosing, rat anti-mouse CSF-1R G2a antibodies were administered IP at $1.5 \mathrm{mg}$ per dose on days 1 and 4. For "Post- $\alpha$ CSF-1R" dosing, rat anti-mouse CSF-1R G2a antibodies were administered IP at $1.5 \mathrm{mg}$ per dose on days 8 and 11 .

\section{Survival studies}

For survival studies, the mice were monitored at least once a day. Mice with signs of distress, including hunched posture and lethargy, were euthanized and considered to have reached the "survival" endpoint in accordance to IACUC guidelines.

\section{Analysis of liver metastasis infiltrating immune cells}

Mice were sacrificed and liver metastasis infiltrating immune cells were harvested on day 14, which was one week after the dose of GVAX. Each liver was mechanically processed and suspended sequentially first through a $100-\mu \mathrm{m}$ nylon filter and then through a $40-\mu \mathrm{m}$ nylon filter, and brought to a volume of $25 \mathrm{~mL}$ in T-cell media. The cell suspensions were centrifuged at $1500 \mathrm{rpm}$ for $5 \mathrm{~min}$. The supernatant was aspirated, and the cell pellets were resuspended in $4 \mathrm{~mL}$ of ACK lysing buffer (Quality Biological). After 2 min in ACK buffer, the lysing was quenched by adding T-cell media up to a volume of $50 \mathrm{~mL}$. The samples were then centrifuged at $1500 \mathrm{rpm}$ for $5 \mathrm{~min}$, and the supernatant was aspirated. The cell pellet was resuspended in $6 \mathrm{~mL}$ of $80 \%$ Percoll (GE Healthcare), then overlaid with $6 \mathrm{~mL}$ of $40 \%$ Percoll, and centrifuged at $3200 \mathrm{rpm}$ for $25 \mathrm{~min}$ without braking. The leukocyte layer was removed and resuspended in $50 \mathrm{~mL}$ of T-cell media, washed twice and re-suspended in PBS. 


\section{Cell surface staining and flow cytometry}

Following the isolation of liver metastasis pancreatic tumor infiltrating immune cells, the cells from mice from the same treatment group were pooled and resuspended in PBS at a concentration of $2 \times 10^{7}$ cells $/ \mathrm{mL}$. For each treatment group, triplicates of this pooled cell suspension were placed into wells of a 96-well plate at a volume of $100 \mu \mathrm{L}$ per well. The cells in each well were stained with Live-Dead Aqua (Invitrogen) for 30 min on ice, washed twice with PBS and then blocked with rat anti-mouse Fc antibody (CD16/CD32, clone 2.4G2, BD Biosciences) in FACs buffer for $10 \mathrm{~min}$ on ice. The FACs buffer consisted of HBSS (Sigma) with $2 \%$ bovine calf serum (Sigma), 0.1\% sodium azide (Sigma) and 0.1\% HEPES. After Fc blocking, the cells were stained for the following anti-mouse fluorophores for $1 \mathrm{~h}$ on ice: CD45 PerCP-Cy5.5 (clone 30-F11, BD Biosciences), CD4 APC-H7 (clone GK1.5, BD Biosciences), CD8a PE/Cy7 (clone 53-6.7, Biolegend), PD-1 FITC (clone RMP1-30, eBioscience), and CD137 APC (clone 17B5, eBioscience) or OX40 APC (clone OX-86, Biolegend).

The cells were then washed twice and resuspended in FACs buffer, and flow cytometry was performed using the Gallios flow cytometer (Beckman Coulter).

\section{Intracellular staining for interferon- $\gamma$ and flow cytometry}

The isolated liver metastasis pancreatic tumor infiltrating immune cells of each mouse were enriched for CD8+ T-cells by negative isolation as per the manufacturer's protocol (Dynabeads Untouched Mouse CD8 cells, Life Technologies). The isolated CD8+ T-cells were incubated with CD3/CD28 stimulation beads (Dynabeads Mouse T-activator CD3/CD28, Life Technologies) according to manufacturer's protocol at 1:1 cell to bead ratio in T-cell media for $7 \mathrm{~h}$ at $37{ }^{\circ} \mathrm{C}$ in $5 \% \mathrm{CO}_{2}$ before GolgiPlug (BD Biosciences) was added at 1:1000 volume ratio, after which the cells were incubated for 5 more hours at $37{ }^{\circ} \mathrm{C}$ in $5 \%$ $\mathrm{CO}_{2}$. After a total incubation time of $12 \mathrm{~h}$, the beads were removed as per manufacturer's protocol and washed 2 times with PBS. The cells from each mouse were kept separate, and were not pooled. The cells were then stained with Live-Dead Aqua (Invitrogen), CD8a PE/Cy7 (clone 53-6.7, Biolegend), PD-1 FITC (clone RMP1-30, eBioscience) and CD137 efluor450 (clone 17B5, eBioscience) as described above. The cells were washed twice with PBS, resuspended and incubated in Fixation/ Permeabilization buffer (eBioscience) for $30 \mathrm{~min}$ at $4{ }^{\circ} \mathrm{C}$. The cells were washed twice with Permeabilization buffer (eBioscience), and then incubated with interferon- $\gamma$ APC (clone XMG1.2, eBioscience) for $30 \mathrm{~min}$ on ice. The cells were washed in twice with Permeabilization buffer and resuspended in FACs buffer. IgGk1 APC isotype control (clone eBRG1, eBioscience) was used for interferon- $\gamma$ (IFN- $\gamma$ ).

\section{Statistical analysis}

For comparisons of cell density and cell density ratios between high and low CSF-1R expression in the human PDAC samples, the mean values were analyzed using the unpaired t-test. Survival analysis in murine experiments were performed using the univariate Cox model. For comparison of cell number, percentage and cytokine expression in murine experiments between groups, the mean values were analyzed using the unpaired one-way ANOVA with Bonferroni $p$-value adjustment for multiple comparisons. All tests were two-tailed, and $P<0.05$ was considered statistically significant.

\section{Results}

\section{High CSF-1R expression after GVAX administration is} associated with an immunosuppressive TME in human PDAC

We first investigated the effect of high versus low CSF-1R expression within the lymphoid aggregates that formed in the human PDAC tumors after a single neoadjuvant dose of GVAX. As anticipated, low CSF-1R expression was associated with high lymphoid to myeloid cell ratio (Fig. 1a and b) and high CD8+ T-cell to CD68 + cell ratio (Fig. 1c). High CSF-1R expression was associated with a higher infiltration of myelomonocytic cells and immature dendritic cells (DCs) into the lymphoid aggregates (Fig. 1d and e), both of which are associated with immune-suppressive environments. [29-31] However, the level of CSF-1R expression did not have an effect on the infiltration of mature DCs (Fig. 1f). These findings suggest that CSF-1R expression is preferentially associated with immunosuppressive myeloid cells in vaccine-primed PDAC and thus a potential target of immunotherapy for PDAC.

Anti-CSF-1R antibody in combination with GVAX and antiPD-1 antibody improves survival in a metastatic PDAC murine tumor model

Given the effects of CSF-1R expression on the human PDAC tumor immune microenvironment after receiving GVAX, we hypothesized that the addition of anti-CSF-1R antibody $(\alpha C S F-1 R)$ to GVAX and anti-PD-1 antibody $(\alpha \mathrm{PD}-1)$ could further improve survival outcomes. We used the previously reported murine model of liver metastatic pancreatic cancer, where the KPC tumor cells were injected into half the spleen on day 0 to generate liver metastases, and this half of the spleen was removed to avoid residual tumor cells external to the liver $[26,28]$. The mice were treated with one dose of GVAX on day 7, and $\alpha \mathrm{PD}-1$ was given on days 7 and 11 (Fig. 2a). We found that beginning $\alpha C S F-1 R$ treatment after GVAX administration did not enhance the anti-tumor effect of GVAX and $\alpha P D-1$ combination therapy in this mouse model (data not shown). Therefore, $\alpha C S F-1 R$ was subsequently dosed in 


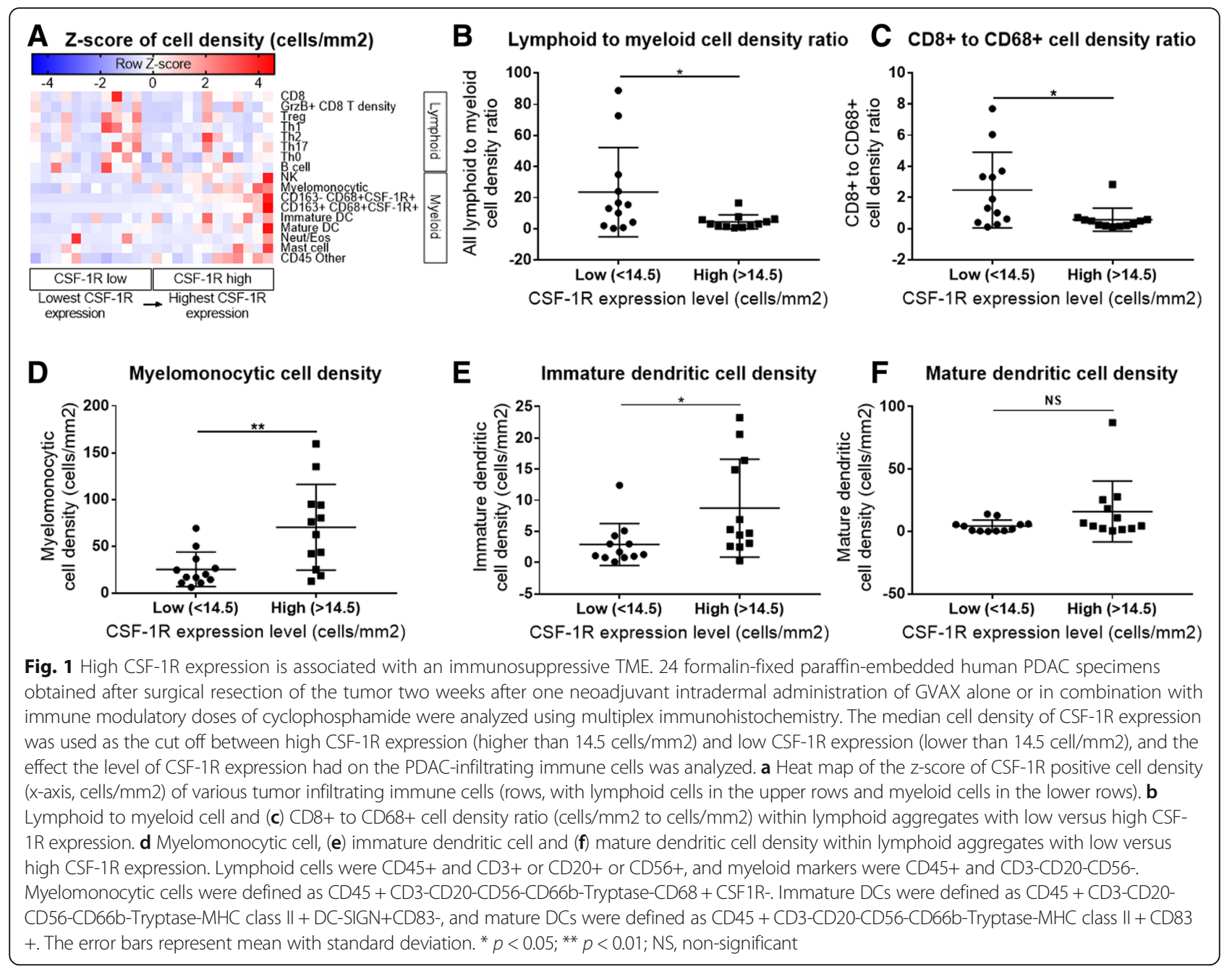

two different schedules to investigate if giving it before GVAX vaccination affected the survival outcome. One group of mice received $\alpha \mathrm{CSF}-1 \mathrm{R}$ on days 1 and 4 prior to GVAX vaccination (regimen called "Pre- $\alpha C S F-1 R$ ", Fig. 2a); and another group of mice received $\alpha C S F-1 R$ on days 1, 4, 8 and 11, which was prior to and after GVAX vaccination (regimen called "Pre/Post- $\alpha$ CSF-1R", Fig. 2a). The combination treatment of GVAX + $\alpha$ PD-1 + Pre/ Post- $\alpha$ CSF-1R improved the day $23 \%$ survival compared to GVAX + $\alpha$ PD- 1 only ( $44 \%$ vs. $0 \%$, HR $0.14,95 \% \mathrm{CI}$, 0.04-0.56, Fig. 2b). There was a trend towards a survival advantage with the GVAX $+\alpha$ PD- $1+$ Pre/Post- $\alpha$ CSF- 1 R treatment regimen over the GVAX $+\alpha P D-1+$ Pre-$\alpha C S F-1 R$ regimen, but this was not statistically significant (44\% vs. $11 \%$, HR $0.31,95 \%$ CI, $0.10-0.98$, Fig. 2b). There was no statistical difference in survival between the GVAX $+\alpha$ PD $-1+$ Pre $-\alpha C S F-1 \mathrm{R}$ and GVAX $+\alpha$ PD- 1 regimens (11\% vs. $0 \%$, HR 0.67, 95\% CI, 0.26-1.73, Fig. 2b). Because only one cycle of GVAX and $\alpha \mathrm{PD}-1$ treatment was given, none of the mice in any of these treatment groups survived beyond day 25 .
Anti-CSF-1R antibody in combination with GVAX and antiPD-1 antibody increases PD-1 + CD137+ T-cells in the TME To understand the mechanistic basis of the enhanced anti-tumor activity of $\alpha$ CSF-1R (Fig. $2 b$ ), we performed flow cytometry analysis of the liver metastasis infiltrating T-cells one week after GVAX administration in the same liver metastatic pancreatic cancer murine model, with day 0 as the day of KPC tumor cell inoculation. All of the treated mice received $\alpha \mathrm{PD}-1$ on days 7 and 11 , and GVAX on day 7 (Fig. 2a). $\alpha C S F-1 R$ was administered in 3 different schedules to investigate its effect on the T-cell population (Fig. 2a), and the mice were sacrificed and the livers harvested on day 14. This analysis demonstrated that the addition of $\alpha \mathrm{CSF}-1 \mathrm{R}$ both before and after the GVAX vaccination not only induced greater numbers of PD-1+CD4+ or CD8+ tumor infiltrating T-cells in the "GVAX + $\alpha$ PD-1 + Pre/Post- $\alpha$ CSF-1R" group (Additional file 1: Figure S1A-D), but also increased the number and percentage of PD-1 + CD137+ T-cells (Fig. 3a-f, Additional file 2: Figure S2D). Up to $10 \%$ of the PD- $1+$ CD $8+$ T-cells expressed CD137 in the 

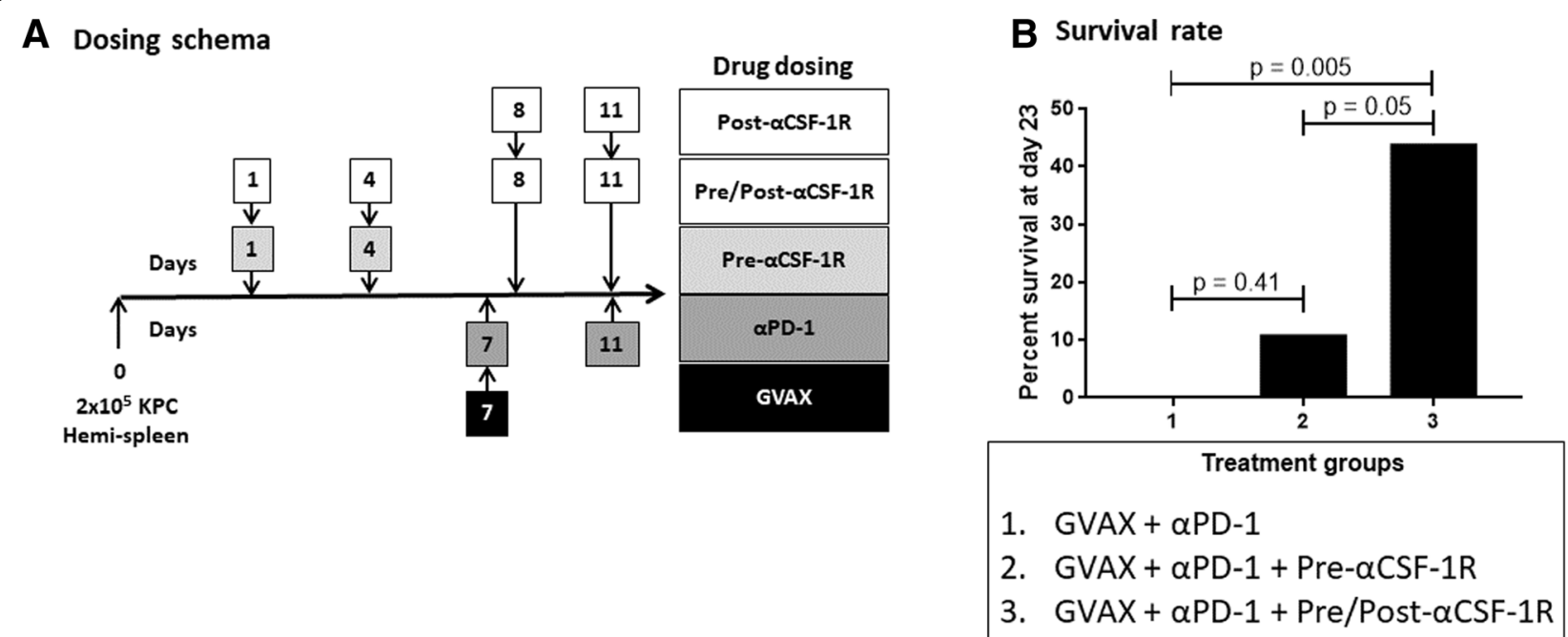

Fig. 2 The addition of aCSF-1R to GVAX therapy and aPD-1 improves survival rate in a liver metastatic pancreatic cancer murine model. a $2 \times 10^{5}$ KPC tumor cells were inoculated via a hemispleen surgery on day 0 , and mice were treated with GVAX and aPD-1 as indicated, and aCSF-1R was dosed in three different ways as shown. b Treated mice were followed for survival, and the percent survival at day 23 was calculated. No mice survived beyond day 25. GVAX + aPD-1 $(n=10)$, GVAX + aPD-1 + Pre-aCSF-1R $(n=9)$, GVAX + aPD-1 + Pre/Post-aCSF-1R $(n=9)$

"GVAX + $\alpha$ PD-1 + Pre/Post- $\alpha$ CSF-1R" group, which was more than 2 or 3 times the average level of expression in the non-treatment and GVAX $+\alpha \mathrm{PD}-1$ groups, respectively (Fig. 3e). The number of infiltrating PD-1 + CD137 + CD4+ T-cells increased by about a factor of 4 (Fig. 3c) and increased by a factor of more than 20 for PD-1+ CD137+ CD8+ T-cells (Fig. 3f) in the "GVAX $+\alpha$ PD-1 + Pre/Post- $\alpha$ CSF-1R" group compared to the GVAX + $\alpha \mathrm{PD}-1$ group. Administering $\alpha \mathrm{CSF}-1 \mathrm{R}$ only before the GVAX vaccination (GVAX + $\alpha$ PD- $1+$ Pre- $\alpha C S F-1 R$ group) had a modest and less robust effect on increasing PD- $1+$ CD137+ T-cells. In contrast, the "GVAX + $\alpha$ PD-1 + Post- $\alpha$ CSF-1R" group did not significantly increase PD-1 + CD137+ T cells compared to the GVAX + $\alpha \mathrm{PD}-1$ group, except for slightly increasing the number of PD-1 + CD137+ CD4+ T-cells (Fig. 3c). These results suggest that $\alpha \mathrm{CSF}-1 \mathrm{R}$ can enhance the expression of the CD137 activation marker in PD-1+ T-cells; however, the administration sequence of $\alpha$ CSF-1R, GVAX and $\alpha$ PD- 1 is important for this activity of $\alpha \mathrm{CSF}-1 \mathrm{R}$.

Anti-CSF-1R antibody in combination with GVAX and antiPD-1 antibody increases PD-1 + OX40+ CD4+ T-cells in the TME

To investigate if the addition of $\alpha C S F-1 \mathrm{R}$ affects other markers of T-cell activation, we also studied OX40 expression in the flow cytometry analysis described above. The addition of $\alpha C S F-1 R$ both before and after GVAX vaccination in the "GVAX $+\alpha$ PD- $1+$ Pre/Post- $\alpha$ CSF-1R" group also increased the number and percentage of PD-1+ OX40+ co-expressing CD4+ T-cells compared to the control groups, non-treatment and GVAX $+\alpha P D-1$ treatment
(Fig. 4a-c). Up to $40 \%$ of PD-1+ CD4+ T-cells expressed OX40+ in the "GVAX + $\alpha$ PD- $1+$ Pre/Post- $\alpha$ CSF- 1 R" group, which is about 2 times higher than the non- $\alpha C S F-1 R$ containing groups (Fig. $4 b$ ). OX40+ expression on CD8+ T-cells was low in all experimental groups, and as such, co-expression of PD-1 and OX40 was low (Fig. 4d-f). Nonetheless, the percentage of PD-1 + OX40+ T-cells amongst CD8+ T-cells was a higher in the "GVAX $+\alpha$ PD- $1+$ Pre/Post- $\alpha$ CSF-1R" group compared to control treatments, non-treatment and GVAX $+\alpha P D-1$ groups, whereas there was no statistically significant difference between the "GVAX + $\alpha$ PD- $1+$ Pre- $\alpha$ CSF-1R" and "GVAX $+\alpha \mathrm{PD}-1+$ Post- $\alpha$ CSF-1R" and the control groups. Amongst PD-1+ CD8+ T-cells however, this slight increase of PD-1 + OX40+ CD8+ T-cells in the "GVAX + $\alpha \mathrm{PD}-1+$ Pre/Post- $\alpha \mathrm{CSF}-1 \mathrm{R}$ " group was not statistically significant from the control groups.

\section{Tumor infiltrating CD8 + PD-1 + CD137+ T-cells express IFN- $\gamma$}

These result suggest that $\alpha \mathrm{CSF}-1 \mathrm{R}$ may convert the PD-1+ exhausted T-cells into activated effector T-cells that express CD137. To examine the activation level of these CD8 + T-cells that co-expressed PD- 1 and CD137, we measured the IFN- $\gamma$ production of these tumor infiltrating CD8 + PD-1 + CD137+ T-cells after isolating CD8+ T-cells and incubating them with $\mathrm{CD} 3 / \mathrm{CD} 28$ stimulation beads, and then performed flow cytometry analysis for CD137, PD-1 and IFN- $\gamma$ expression amongst these CD8+ T-cells. Most of the CD8 + PD-1 + CD137+ T-cells (median about 80$90 \%$ ) in the treated, as well as untreated groups, expressed IFN- $\gamma$ (Fig. 5a). Interestingly, a higher percentage of the 


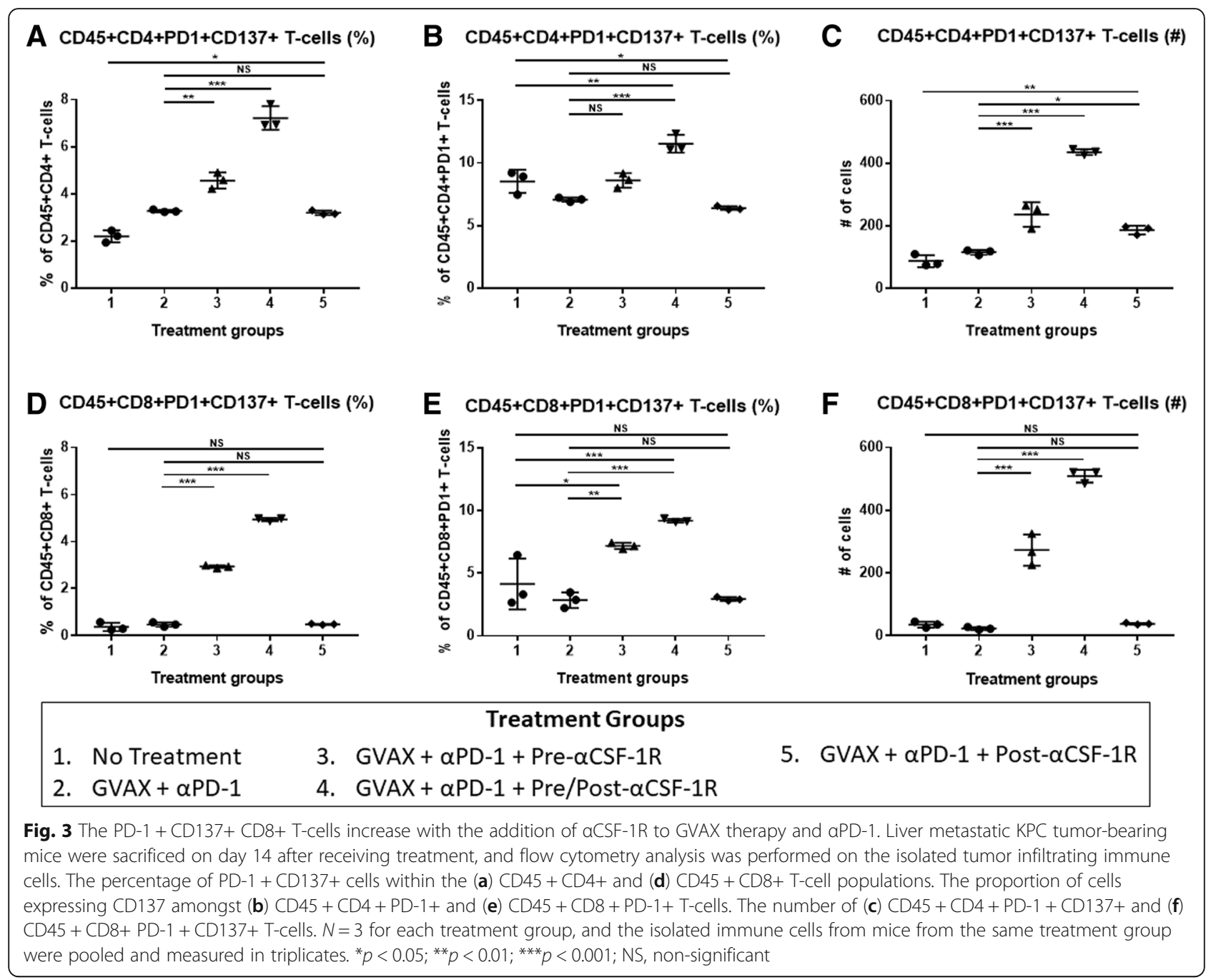

CD8 + PD-1 + CD137+ T-cells expressed IFN- $\gamma$ compared to the CD8 + PD-1 + CD137- T-cells (Fig. 5b), suggesting that $\mathrm{CD} 8+\mathrm{PD}-1+\mathrm{CD} 137-\mathrm{T}$-cells, which may express other activation markers, are less effective.

\section{Discussion}

In this report, we found that higher expression of CSF-1R is associated with a higher intratumoral myeloid to lymphoid ratio, which is associated with poorer survival in PDAC patients who received GVAX. Interestingly, this higher CSF-1R expression is associated with a greater intratumoral infiltration of immature DCs, but not mature DCs. As DCs fail to mature and activate T-cells, these T-cells can become tolerogenic or anergic. Hence, low CSF-1R expression leads to higher infiltration of lymphoid cells (including CD8+ T-cells), and lower infiltration of certain myeloid cell subsets associated with poor prognosis, while sparing the mature DCs that are important for antigen presentation in vaccine therapies. These findings suggest that CSF-1R, in conjunction with cancer vaccine therapy, could be targeted to deplete immunosuppressing tumor infiltrating myeloid cells and modulate the remaining myeloid cells towards an anti-tumor phenotype. This study subsequently demonstrated in a preclinical model that anti-CSF-1R antibody therapy enhanced the antitumor activity of the combination of GVAX and anti-PD-1 antibody. The anti-tumor efficacy of anti-CSF-1R antibody appears to be partially achieved by its role in converting PD-1+ exhausted T-cells into CD137 + activated T-cells in the PDAC tumors. Accordingly, we identified a subpopulation within both CD4+ and CD8+ T-cells that expressed both PD-1 and CD137. The increase in expression of CD137 after therapy that included anti-CSF-1R, compared to treatment that did not, was more robust within PD-1+ CD8+ T-cells than PD-1+ CD4 + T-cells. The vast majority of these PD-1 + CD137+ CD8+ T-cells expressed IFN- $\gamma$, and thus were functional effector T-cells. Furthermore, combination immunotherapy including anti-CSF-1R also increased OX40 expression amongst PD-1+ CD4+ T-cells, though there was minimal expression of OX40 on PD-1+ CD8+ T-cells. 

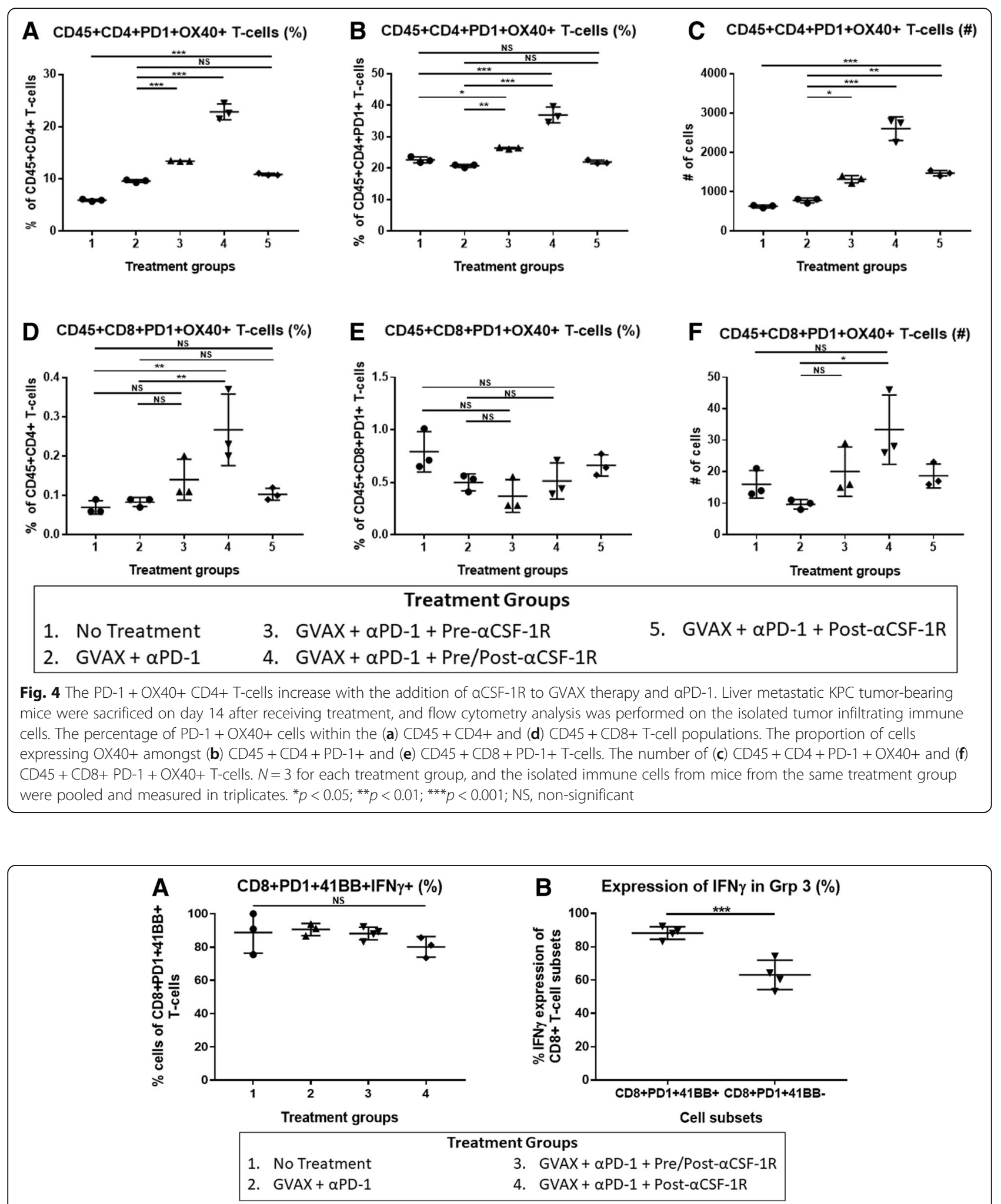

Fig. 5 CD8+ T-cells that co-express PD-1 and CD137 express IFN- $\gamma$. Liver metastatic KPC tumor-bearing mice were sacrificed on day 14 after receiving treatment, and flow cytometry analysis was performed on the isolated tumor infiltrating CD8+ T-cells after incubation with CD3/CD28 stimulation beads. a IFN- $\gamma$ production of CD8 + PD-1 + CD137+ T-cells. b IFN- $\gamma$ production of CD8 + PD-1 + CD137+ and CD8 + PD-1 + CD137- Tcells in the GVAX + aPD-1 + Pre/Post-aCSF-1R group. N=3-4 for each treatment group, and each data point represents cells from one mouse. The error bars represent mean with standard deviation. ${ }^{* * *} p<0.001$; NS, non-significant 
CD137 and OX40 are members of the tumor necrosis factor receptor (TNFR) superfamily, and are involved in T-cell expansion and prevention of antigen-induced cell death [32]. They are thereby responsible for enlarging the memory T-cell population, which is essential to maintaining the immune protection conferred by vaccinations [33]. It was previously thought that there was a dichotomy in roles between these two members of the TNFR superfamily, such that OX40 played a more central function for CD4+ $\mathrm{T}$-cell expansion and survival, and $41 \mathrm{BB}$ played a similar function for CD8+ T-cells, and to some extent, our findings may reflect this. [33-35] However, subsequent studies suggest that these receptors have roles in both cell types, and that their expression is dependent on the immune environment and timing from initial T-cell priming. [36, 37] It has been shown that CD137 expression on CD8+ T-cells persists for a longer time, whereas OX40 expression is transient [37]. As such, it is possible that this combination treatment with anti-CSF-1R either does not induce OX40 expression on PD-1+ CD8+ T-cells, or the expression of OX40 was too transient and subsequently not measured.

The PD-1 + CD137+ T-cells were found in the PDAC tumors of all the mice, regardless of whether they were treated by GVAX, anti-PD-1 or anti-CSF-1R antibody. Although PD1 + CD137+ T-cells are only a small percentage among all the T-cells, it is possible that PD-1+ T-cells expressing other activation markers exist. More importantly, as the majority of the PD-1 + CD137+ T-cells have the capacity of expressing IFN- $\gamma$, even if this subpopulation of T-cells is small, they may still possess a strong anti-tumor effector T-cell function. Indeed, a small percentage of CD3 + CD8+ T-cells that are PD- $1+\mathrm{CD} 137+(0.6$ to $5.1 \%)$ have been found in some patients with metastatic melanoma and appear to have the ability to recognize neoantigens [38].

It remains to be explored how anti-CSF-1R antibody leads to the significant increase in the percentage and number of intratumoral PD-1+ CD4+ and PD-1+ CD8+ T-cells that express CD137 and PD- $1+$ CD4+ T-cells that express OX40. As CD137 expression represents T-cell activation during the T-cell priming process, it is possible that the effect of anti-CSF-1R on myeloid cells accounted for this modulation of T-cell priming. Anti-CSF-1R antibody has been reported to spare the activated antigen-presenting DCs from depletion from the TME [20, 21, 39]. This study supports the rational that targeting tumor infiltrating myeloid cells can activate T-cells with exhausted phenotypes, but the survival advantage of the combination therapy with $\alpha$ CSF-1R is still limited. The limited efficacy is likely partly due CSF-1R being a pan-myeloid marker, and thus a more specific agent targeting immunosuppressive myeloid cells may improve the efficacy.

Interestingly, the sequence of anti-CSF-1R antibody and the treatment of anti-PD-1 antibody and GVAX appears to be critical. Having anti-CSF1R antibody both before and after the GVAX treatment yields the largest survival benefit and the largest increase in PD- $1+$ CD137+ T-cells and PD-1 + OX40+ CD4+ T-cells. However, in human studies, such a combination of anti-PD-1 antibody, GVAX, and anti-CSF-1R antibody would be administered as multiple cycles. When they are given for multiple cycles, the differential effect of the sequencing of anti-CSF-1R with anti-PD-1 and GVAX treatment may be abrogated, and the effects of multiple cycles of therapy will be investigated in future studies. Nevertheless, anti-tumor efficacy of the combination of anti-PD-1 antibody, GVAX and anti-CSF1R antibody warrants further investigation in human studies for PDAC.

\section{Conclusions}

In this study, we demonstrate that the level of expression of CSF-1R within the lymphoid aggregates induced by GVAX affects the tumor immune microenvironment; high CSF-1R expression is associated with an immunosuppressive environment in patients who received GVAX. To counteract the immunosuppressing effects of CSF-1R expression within the TME, we examined the combination therapy of GVAX vaccine, and anti-PD-1 and anti-CSF-1R antibodies in a murine model of metastatic PDAC. This combination therapy improved the survival rate, increased the percentage of $\mathrm{CD} 4+$ and CD8+ T-cells that co-expressed PD-1 and CD137 and increased the percentage of PD-1 + OX40+ CD4+ T-cells within the tumors. The PD-1+CD137+ CD8+ T-cells had high IFN- $\gamma$ expression, suggesting that these cells in part were responsible for the improved anti-tumor activity of the combination therapy. Our results demonstrate that adding a myeloid-targeting agent to vaccine-based cancer immunotherapy can reverse the anergy of intra-tumoral T-cells in immune-quiescent tumors. The findings in this study support the clinical evaluation of this combination therapy in patients with PDAC.

\section{Additional files}

Additional file 1: Figure S1. PD-1 expression on CD4+ and CD8+ T cells can increase with the addition of aCSF-1R to GVAX therapy and aPD-1. Liver metastatic KPC tumor-bearing mice were sacrificed on day 14 after receiving treatment, and flow cytometry analysis was performed on the isolated tumor infiltrating immune cells. PD-1 expression within the (A) CD45 + CD4+ T-cell and (C) CD45 + CD8+ T-cell populations. The number of (B) CD45 + CD4 + PD-1+ and (D) CD45 + CD8 + PD-1+ T-cells. $N=3$ for each treatment group, and the cells from the mice from the same treatment group were pooled and measured in triplicates. ${ }^{*} p<0.05$; ${ }^{* *} p<0.01$; ${ }^{* *} p<0.001$; NS, non-significant. (PNG $32 \mathrm{~kb}$ )

Additional file 2: Figure S2. PD-1 + CD137+ expression increases when aCSF-1R is administered before and after GVAX vaccination in combination with aPD-1. Representative flow cytometry dot plots of PD-1 and CD137 expression amongst CD8+ and CD4+ T-cells between the different treatment regimens containing aCSF-1R, GVAX and aPD-1. (PNG 269 kb) 


\section{Abbreviations}

Cl: Confidence interval; CSF-1: Colony stimulating factor-1; CSF-1R: Colony stimulating factor-1 receptor; DC: Dendritic cell; DC-SIGN: Dendritic CellSpecific Intercellular adhesion molecule-3-Grabbing Non-integrin; GMCSF: Granulocyte-macrophage colony-stimulating factor; HR: hazard ratio; IHC: Immunohistochemistry; MDSC: Myeloid-derived suppressor cell; PD1: Programmed death-1; PDAC: Pancreatic ductal adenocarcinoma; TAM: Tumor-associated macrophage; TME: Tumor microenvironment; TNFR: Tumor necrosis factor receptor; aCSF-1R: Anti-colony stimulating factor-1 receptor antibody; aPD-1: Anti-programmed death-1 antibody

\section{Acknowledgements}

We wish to thank Dr. Todd Armstrong for helpful discussions. We also thank Abigail Brittingham for technical help.

\section{Funding}

This work was supported in part by the ASCO Young Investigator Award (M.T.S.), NIH T32 CA 9071-36 (M.T.S.), OCTRI \#UL1TR000128 from NCATS/NIH (T.T., L.M.C.), Grant-in-Aid for Scientific Research $17 \mathrm{H} 07016$ from the Japan Society for the Promotion of Science (T.T.), a BMS II-ON grant (L.Z.), NIH grant R01 CA169702 (L.Z.), NIH grant R01 CA197296 (L.Z.), the Viragh Foundation and the Skip Viragh Pancreatic Cancer Center at Johns Hopkins (L.Z.,E.M.J.), National Cancer Institute Specialized Programs of Research Excellence in Gastrointestinal Cancers grant P50 CA062924 (L.Z..E.M.J.) and Sidney Kimmel Comprehensive Cancer Center grant P30 CA006973 (L.Z.,E.M.J.).

\section{Availability of data and materials}

The data used and/or analyzed for this study is available from the corresponding author at reasonable request.

\section{Authors' contributions}

MTS performed the statistical analysis of the effect of CSF-1R expression in the human PDAC specimens. TT performed the multiplex immunohistochemistry of the human specimens, with contributions from LC. MTS designed and analyzed the murine experiments, with contribution from LZ. MTS performed the murine experiments with help from SM, DLT and ABB. DD provided input and advice for statistical analysis. MTS wrote the manuscript, with contributions from LZ and EMJ. All authors read and approved the final manuscript.

\section{Ethics approval and consent to participate}

Institutional review board (IRB) approval was obtained before initiation of the clinical trial. As this was a retrospective study, IRB approved the request for waiver for patients' consents. All animal studies were approved by the Institutional Animal Care and Use Committee (IACUC).

\section{Consent for publication}

Not applicable

\section{Competing interests}

L.Z. receives grant supports from Bristol-Meyer Squibb, Merck, iTeos, Amgen, Gradalis, and Halozyme, and receives the royalty for licensing GVAX to Aduro Biotech. L.Z. is a paid consultant/Advisory Board Member at Biosynergies, Oncorus, Alphamab, Merck, NovaRock and AstroZeneca. E.M.J. receives the royalty for licensing GVAX to Aduro Biotech.

\section{Publisher's Note}

Springer Nature remains neutral with regard to jurisdictional claims in published maps and institutional affiliations.

\section{Author details}

'Department of Oncology, Johns Hopkins University School of Medicine, Baltimore, MD, USA. ${ }^{2}$ The Sidney Kimmel Cancer Center, Johns Hopkins University School of Medicine, Baltimore, MD, USA. ${ }^{3}$ Department of Surgery, Johns Hopkins University School of Medicine, Baltimore, MD, USA. ${ }^{4}$ Department of Otolaryngology-Head and Neck Surgery, Kyoto Prefectural University of Medicine, Kyoto, Japan. ${ }^{5}$ Department of Cell, Developmental \& Cancer Biology, Oregon Health and Science University, Portland, OR, USA. ${ }^{6}$ Knight Cancer Institute, Oregon Health and Science University, Portland, OR, USA. ${ }^{7}$ The Skip Viragh Center for Pancreatic Cancer Research and Clinical Care, Johns Hopkins University School of Medicine, Baltimore, MD, USA. ${ }^{8}$ Pancreatic Cancer Precision Medicine Program, Johns Hopkins University
School of Medicine, Baltimore, MD, USA. ${ }^{9}$ The Bloomberg-Kimmel Institute for Cancer Immunotherapy, Johns Hopkins University School of Medicine, Baltimore, MD, USA.

Received: 10 September 2018 Accepted: 26 October 2018

Published online: 13 November 2018

\section{References}

1. Siegel RL, Miller KD, Jemal A. Cancer statistics, 2018. CA Cancer J Clin. 2018; 68(1):7-30.

2. Brahmer JR, Tykodi SS, Chow LQ, et al. Safety and activity of anti-PD-L1 antibody in patients with advanced cancer. N Engl J Med. 2012;366(26):2455-65.

3. Lutz ER, Wu AA, Bigelow E, et al. Immunotherapy converts nonimmunogenic pancreatic tumors into immunogenic foci of immune regulation. Cancer Immunol Res. 2014;2(7):616-31.

4. Le DT, Wang-Gillam A, Picozzi V, et al. Safety and survival with GVAX pancreas prime and listeria monocytogenes expressing mesothelin (CRS207) boost vaccines for metastatic pancreatic cancer. J Clin Oncol. 2015; 33(12):1325-33.

5. Fu J, Kanne DB, Leong M, Glickman LH, et al. STING agonist formulated cancer vaccines can cure established tumors resistant to PD-1 blockade. Sci Transl Med. 2015:7(283):283-52.

6. Fukunaga A, Miyamoto M, Murakami S, et al. CD8+ tumor-infiltrating lymphocytes together with CD4+ tumor-infiltrating lymphocytes and dendritic cells improve prognosis of patients with pancreatic adenocarcinoma. Pancreas. 2004:28(1):e25-31.

7. Vonderheide RH, Bayne $\sqcup$. Inflammatory networks and immune surveillance of pancreatic carcinoma. Curr Opin Immunol. 2013;25(2):200-5.

8. Clark CE, Hingorani SR, Mick R, Combs C, Tuveson DA, Vonderheide RH. Dynamics of the immune reaction to pancreatic cancer from inception to invasion. Cancer Res. 2007;67(19):9518-27.

9. Jaffee EM, Schutte M, Gossett J, et al. Development and characterization of a cytokine-secreting pancreatic adenocarcinoma vaccine from primary tumors for use in clinical trials. Cancer J Sci Am. 1998;4(3):194-203.

10. Jaffee EM, Hruban RH, Biedrzycki B, et al. Novel allogeneic granulocytemacrophage colony-stimulating factor-secreting tumor vaccine for pancreatic cancer: a phase I trial of safety and immune activation. J Clin Oncol. 2001;19(1):145-56.

11. Le DT, Lutz E, Uram JN, et al. Evaluation of ipilimumab in combination with allogeneic pancreatic tumor cells transfected with a GM-CSF gene in previously treated pancreatic cancer. J Immunother. 2013;36(7):382-9.

12. Dieu-Nosjean MC, Antoine M, Danel C, et al. Long-term survival for patients with non-small-cell lung cancer with intratumoral lymphoid structures. J Clin Oncol. 2008:26(27):4410-7.

13. Takahiro T, Kumar S, Borkar RN, et al. Quantitative multiplex immunohistochemistry reveals myeloid-inflamed tumor-immune complexity associated with poor prognosis. Cell Rep. 2017;19(1):203-17.

14. DeNardo DG, Brennan DJ, Rexhepaj E, et al. Leukocyte complexity predicts breast cancer survival and functionally regulates response to chemotherapy. Cancer Discov. 2011;1(1):54-67.

15. Ruffell B, Coussens LM. Macrophages and therapeutic resistance in cancer. Cancer Cell. 2015;27(4):462-72.

16. Bailey P, Chang DK, Nones K, et al. Genomic analyses identify molecular subtypes of pancreatic cancer. Nature. 2016;531:47-52.

17. Knudsen ES, Vail P, Balaji U, et al. Stratification of pancreatic ductal adenocarcinoma: combinatorial genetic, stromal, and immunologic markers. Clin Cancer Res. 2017;23(15):4429-40

18. Dai XM, Ryan GR, Hapel AJ, et al. Targeted disruption of the mouse colonystimulating factor 1 receptor gene results in osteopetrosis, mononuclear phagocyte deficiency, increased primitive progenitor cell frequencies, and reproductive defects. Blood. 2002;99(1):111-20.

19. Ries $\mathrm{CH}$, Cannarile MA, Hoves $\mathrm{S}$, et al. Targeting tumor-associated macrophages with anti-CSF-1R antibody reveals a strategy for Cancer therapy. Cancer Cell. 2014;25(6):846-59.

20. Zhu Y, Knolhoff BL, Meyer MA, et al. CSF1/CSF1R blockade reprograms tumor-infiltrating macrophages and improves response to T-cell checkpoint immunotherapy in pancreatic Cancer models. Cancer Res. 2014;74(18):5057-69.

21. Candido JB, Morton JP, Bailey P, et al. CSF1R+ macrophages sustain pancreatic tumor growth through T cell suppression and maintenance of key gene programs that define the squamous subtype. Cell Rep. 2018;23(5): 1448-60. 
22. Peranzoni E, Lemoine J, Vimeux $L$, et al. Macrophages impede CD8 T cells from reaching tumor cells and limit the efficacy of anti-PD-1 treatment. Proc Natl Acad Sci U S A. 2018;115(17):E4041-50.

23. Eissler $N$, Mao $Y$, Brodin $D$, et al. Regulation of myeloid cells by activated $T$ cells determines the efficacy of PD-1 blockade. Oncoimmunology. 2016; 5(12):e1232222.

24. Cannarile MA, Weisser M, Jacob W, et al. Colony-stimulating factor 1 receptor (CSF1R) inhibitors in cancer therapy. J Immunother Cancer. 2017; 5(1):53.

25. Wainberg Z, Piha-Paul S, Luke J, et al. O42 First-in-human phase 1 dose escalation and expansion of a novel combination, anti-CSF-1 receptor (cabiralizumab) plus anti-PD-1 (nivolumab), in patients with advanced solid tumors. Oral presentation at: 32nd Annual Meeting and Pre-Conference Programs of the Society for Immunotherapy of Cancer (SITC 2017); November 8-12, 2017: National Harbor, MD.

26. Soares KC, Rucki AA, Wu AA, et al. PD-1/PD-L1 blockade together with vaccine therapy facilitates effector $T$ cell infiltration into pancreatic tumors. J Immunother. 2015;38(1):1-11.

27. Hingorani SR, Wang L, Multani AS, et al. Trp53R172H and KrasG12D cooperate to promote chromosomal instability and widely metastatic pancreatic ductal adenocarcinoma in mice. Cancer Cell. 2005;7(5):469-83.

28. Soares KC, Foley K, Olino K, et al. A preclinical murine model of hepatic metastases. J Vis Exp. 2014;91:e51677.

29. Ostrand-Rosenberg S, Sinha P, Beury DW, Clements VK. Cross-talk between myeloid-derived suppressor cells (MDSC), macrophages, and dendritic cells enhances tumor-induced immune suppression. Semin Cancer Biol. 2012; 22(4):275-81.

30. Gabrilovich D. Mechanisms and functional significance of tumour-induced dendritic-cell defects. Nat Rev Immunol. 2004;4(12):941-52.

31. Poschke I, Mao Y, Adamson L, Salazar-Onfray F, Masucci G, Kiessling R. Myeloid-derived suppressor cells impair the quality of dendritic cell vaccines. Cancer Immunol Immunother. 2012;61(6):827-38.

32. Song A, Tang X, Harms KM, Croft M. OX40 and BCl-XL promote the persistence of CD8 T cells to recall tumor-associated antigen. J Immunol. 2005;175(6):3534-41.

33. Munks MW, Mourich DV, Mittler RS, Weinberg AD, Hill AB. 4-1BB and OX40 stimulation enhance CD8 and CD4 T-cell responses to a DNA prime, poxvirus boost vaccine. Immunology. 2004;112(4):559-66.

34. Wojciech D, Bertram EM, Sharpe AH, Watts TH. 4-1BB and OX40 act independently to facilitate robust CD8 and CD4 recall responses. J Immunol. 2004;173(10):5944-51.

35. Sugamura K, Ishii N, Weinberg AD. Therapeutic targeting of the effector Tcell co-stimulatory molecule OX40. Nat Rev Immunol. 2004;4(6):420-31.

36. Croft M, So T, Duan W, Soroosh P. The significance of OX40 and OX40L to T-cell biology and immune disease. Immunol Rev. 2009;229(1):171-91.

37. Lee SW, Park Y, Song A, Cheroutre H, Kwon BS, Croft M. Functional dichotomy between OX40 and 4-1BB in modulating effector CD8 T cell responses. J Immunol. 2006;177(7):4464-72.

38. Parkhurst M, Gros A, Pasetto A, et al. Isolation of T-cell receptors specifically reactive with mutated tumor-associated antigens from tumor-infiltrating lymphocytes based on CD137 expression. Clin Cancer Research. 2017;23(10): 2491-505.

39. Mitchem JB, Brennan DJ, Knolhoff BL, et al. Targeting tumor-infiltrating macrophages decreases tumor-initiating cells, relieves immunosuppression, and improves chemotherapeutic responses. Cancer Res. 2013;73(3):1128-41.

Ready to submit your research? Choose BMC and benefit from:

- fast, convenient online submission

- thorough peer review by experienced researchers in your field

- rapid publication on acceptance

- support for research data, including large and complex data types

- gold Open Access which fosters wider collaboration and increased citations

- maximum visibility for your research: over $100 \mathrm{M}$ website views per year

At BMC, research is always in progress.

Learn more biomedcentral.com/submissions 